\title{
Association analysis between $H F M 1$ variations and idiopathic azoospermia or severe oligozoospermia in Chinese Men
}

\author{
Wenxiang Zhang ${ }^{1,2}$, Xiaomin Song ${ }^{2}$, Feng $\mathrm{Ni}^{2}$, Jinbao Cheng ${ }^{2}$, Bai-Lin $\mathrm{Wu}^{3,4^{*}}$ \\ \& Hong Jiang ${ }^{1,2^{*}}$ \\ ${ }^{1}$ Clinical College of PLA Affiliated Anhui Medical University, Hefei 230031, China; \\ ${ }^{2}$ Reproductive Medicine Center, the $105^{\text {th }}$ hospital of PLA, Hefei 230031, China; \\ ${ }^{3}$ Children's Hospital of Fudan University; Institute of Biomedical Science, Shanghai Medical College of Fudan University, \\ Shanghai, China 200032; \\ ${ }^{4}$ Boston Children's Hospital and Harvard Medical School, Boston, MA 02115, USA
}

Received August 19, 2016; accepted August 23, 2016; published online November 24, 2016

Citation: Zhang, W., Song, X., Ni, F., Cheng, J., Wu, B.L., and Jiang, H. (2017). Association analysis between HFM1 variations and idiopathic azoospermia or severe oligozoospermia in Chinese Men. Sci China Life Sci 59, 315-318. doi: 10.1007/s11427-016-0274-9

Dear Editor,

Infertility affects $10 \%-15 \%$ of couples trying to conceive, with male factors contributing to as many as $50 \%$ of infertile cases (Meng et al., 2015). Azoospermia or severe oligozoospermia (oligo/azoospermia), clinically characterized by a complete or partial absence of sperm in the ejaculate, account for $10 \%-15 \%$ of male infertility (Wosnitzer et al., 2014). Approximately $20 \%-30 \%$ of men with oligo/azoospermia are attributed to the known genetic factors, including chromosomal and single gene alterations, while up to $80 \%$ of idiopathic oligo/azoospermia remain unknown and further research is required to identify candidate gene(s) (Stahl et al., 2012).

A number of genes involved in spermatogenesis have been identified being related to idiopathic oligo/azoospermia, such as NPAS2 (Ramasamy et al., 2015). HFM1 (Helicase Family Member 1), an ATP-dependent DNA helicase homolog, encodes a meiosis-specific protein necessary for homologous recombination of chromosomes, exhibited dimorphism in gametogenesis (Hawley et al., 2013). The $\mathrm{Hfml}$ null mice were infertile for both sexes, of which males showed meiotic arrest at the end of prophase I. The

*Corresponding author (email: bai-lin.wu@childrens.harvard.edu; jiangh105@sina.com) phenotypes of $\mathrm{Hfml}^{-/}$males were similar to the clinical characteristics of men with oligo/azoospermia (Hawley et al., 2013). Compound heterozygous mutations in HFMI gene (c.1686-1G>C and c.2651T>G/p.I884S, c.2206G $>$ A/ p.G736S and c.3929_3930 delinsG/p.P1310R fs*41) were identified in women with familial or sporadic primary ovarian insufficiency (POI) in our previous study, suggesting that biallelic mutations in $H F M 1$ can result in recessive POI in humans (Wang et al., 2014). However, whether HFMI is involved in male infertility is still unknown. The aim of this study is to investigate the association of HFMI gene variants with the idiopathic oligo/azoospermia in Chinese men.

Mutational analysis was performed for 80 Chinese patients with idiopathic oligo/azoospermia and 160 healthy controls (matched for ethnic backgrounds and ages) by direct sequencing of the coding regions in HFMI gene. Seven variations (six were singleton and one was detected twice, summarized in Table 1) were found in five unrelated patients. Among of them, four novel variations (c.437C $>\mathrm{A} /$ p.Thr146Lys, c.1328A>G/p.Lys443Arg, c.1481T>C/p. Val494Ala and c.3689C $>$ G/p.Ser1230Cys) were absent in 160 matched controls and population data from the public available databases (the dbSNP, the 1000 Genomes Project, the NHLBI-ESP, and the ExAC, summarized in Table 1). The variation rate of $H F M 1$ in oligo/azoospermia group was significantly higher than matched controls and database 


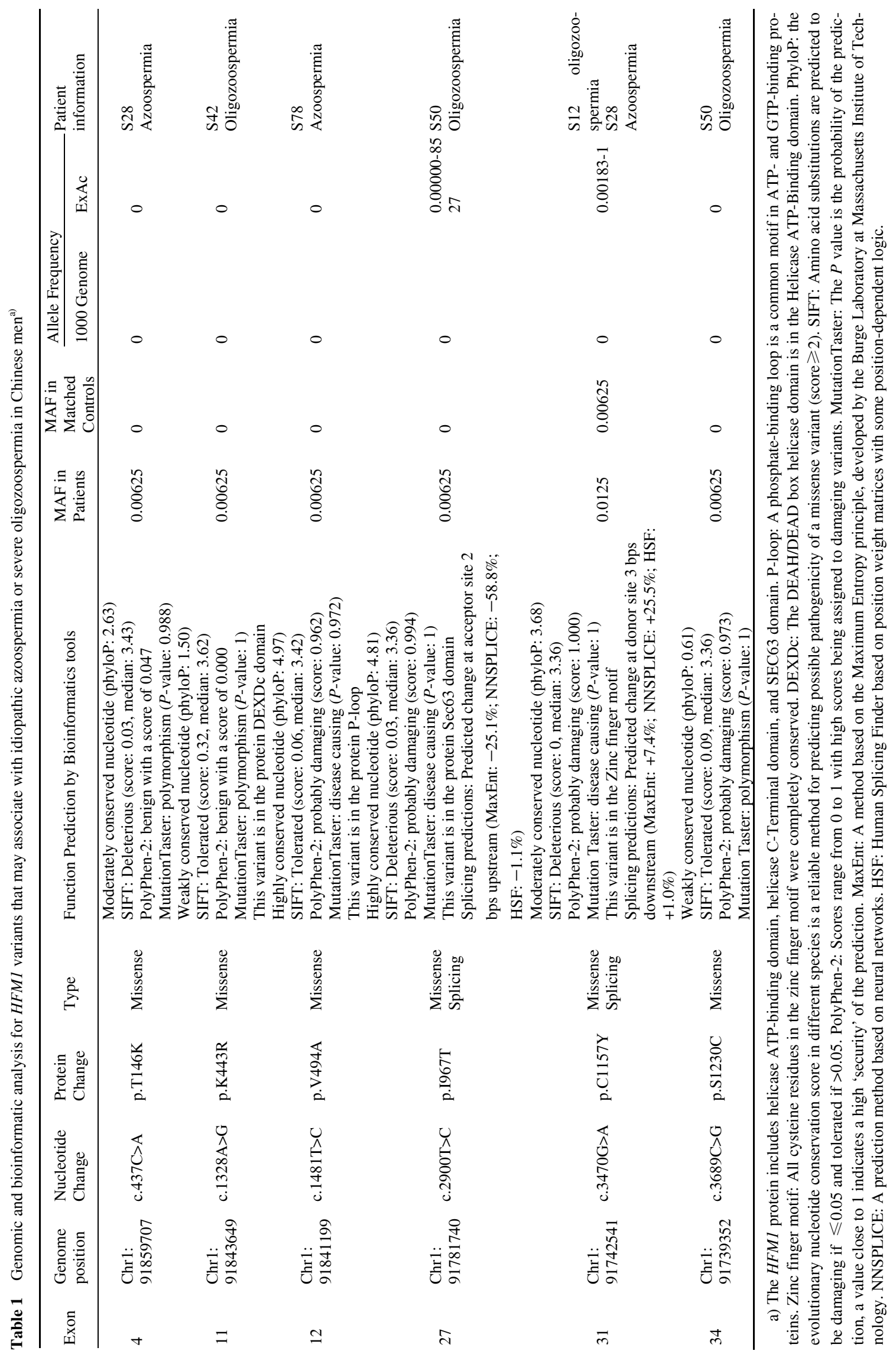


control group $(P<0.008$ and $P<0.00001$, respectively). Two novel variants were identified locating in protein domains (p.Val494Ala in P-loop and p.Lys443Arg in the DEAH/ DEAD box helicase domain (DEXDc)). The former was predicted deleterious to $H F M 1$ protein function and the later was predicted benign by SIFT, Polyphen- 2 and Mutation Taster. These two compound heterozygous variants were identified in two unrelated patients. Of them, one patient had a novel missense variant c.437C >A/p.Thr146Lys (predicted to be deleterious by SIFT) and a known missense variant c.3470G >A/p.Cys1157Tyr in zinc finger motif (predicted to be disease causing by SIFT, Polyphen-2 and MutationTaster, and affecting splicing sites by MaxEnt, NNSPLICE and HSF). The other patient had a novel missense variant c.3689C $>$ G/p.Ser1230Cys (predicted to be probably damaging on the structure and/or function of the HFM1 protein by PolyPhen-2) and a rare known variant c.2900T>C/p.Ile967Thr in SEC63 domain (predicted to be disease causing by SIFT, Polyphen- 2 and Mutation Taster, and affecting splicing sites by MaxEnt, NNSPLICE and HSF). These results indicate that HFM1 gene variants may associate with idiopathic oligo/azoospermia in Chinese male individuals.

HFM1 (the human homologue of yeast Mer3), a meiotic gene comprised of 39 exons mapped to human chromosome $1 \mathrm{p} 22.2$ and specifically expressed in germ-line tissues such as testis and ovary, is necessary for normal progression of homologous recombination and proper synapsis between homologous chromosomes during meiosis process in many organisms (Tanaka et al., 2006; Wang et al., 2009). Hawley et al. (2013) had demonstrated that the absence of HFMI protein would result in deficient recombination and incomplete meiosis-specific modification of chromosome structure of $\mathrm{Hfml}^{-\mathrm{I}_{-}}$mouse model. $\mathrm{Hfml} \mathrm{I}^{-/-}$adult mice presented no overt somatic phenotype apart from the lack of reproductive function. The phenotypes of $\mathrm{Hfml}$ knockout female mice include a significant reduction in ovary size, reduced number of follicles and corpora lutea, and an increase in stromal cells, which were similar to the phenotypes of POI patients (Hawley et al., 2013; Wang et al., 2014). As demonstrated in our previous study, mutations in HFMI gene could result in recessive POI (Wang et al., 2014).

Since the phenotype of meiotic mouse mutants commonly show sexual dimorphism (Hawley et al., 2013; Li et al., 2002), the aim of this study is to validate HFM1 as a candidate gene for idiopathic oligo/azoospermia. Here for the first time we demonstrated that HFMI gene variants may associate with idiopathic oligo/azoospermia. The HFM1 protein mainly includes helicase ATP-binding domain, helicase C-Terminal domain, and SEC63 domain (Figure S1 in Supporting Information). Four out of six variants found in this study located within the functionally important domains: DEXDc, one of the super family of helicases could unwind DNA and/or RNA, located in the Helicase ATP-Binding domain; the phosphate-binding loop (p-loop), a common motif in ATP- and GTP-binding proteins, containing nucleoside triphosphate hydrolase is involved in the general folding of protein molecules; the SEC63 domain is required for assembly of functional endoplasmic reticulum translocons; the Zinc finger motif, in which all cysteine residues in the zinc finger motif were completely conserved (Saraste et al., 1990; Tanaka et al., 2006). In addition, the p.Ile967Thr located in SEC63 domain might result in an alteration at splice acceptor site 2 bps upstream in exon 27, while the p.Cys1157Tyr located in zinc finger motif might lead to a change at splice donor site 3 bps downstream in exon 31 . These two variants involved in splicing sites probably lead to $H F M 1$ protein dysfunction.

In conclusion, oligo/azoospermia as a complex disease is characterized by genetic heterogeneity with ethnics (Stahl et al., 2012). Our study suggested that HFM1 gene variants may be associated with idiopathic azoospermia or severe oligozoospermia in Chinese men. Additional study with more patients and functional assays would be valuable for not only confirming the association but also further exploring the biological mechanisms of HFMI variations in the spermatogenesis impairment.

Compliance and ethics The author(s) declare that they have no conflict of interest.

Acknowledgements This work was supported by the National Natural Science Foundation of China (81501216), and Anhui Provincial Natural Science Foundation (1608085MH165). This work was also supported in part by the National Basic Research Program of China grants from the Ministry of Science and Technology of China (2013CB945404 and 2010CB529601).

Hawley, R.S., Guiraldelli, M.F., Eyster, C., Wilkerson, J.L., Dresser, M.E., and Pezza, R.J. (2013). Mouse hfm1/mer3 is required for crossover formation and complete synapsis of homologous chromosomes during meiosis. PLoS Genetics 9, e1003383.

Li, Y., Liu, J.G., Hoja, M.R., Wilbertz, J., Nordqvist, K., and Hoog, C. (2002). Female germ cell aneuploidy and embryo death in mice lacking the meiosis-specific protein scp3. Science 296, 1115-1118.

Meng, Q., Ren, A., Zhang, L., Liu, J., Li, Z., Yang, Y., Li, R., and Ma, L. (2015). Incidence of infertility and risk factors of impaired fecundity among newly married couples in a chinese population. Reprod Biomed online 30, 92-100.

Ramasamy, R., Bakircioglu, M.E., Cengiz, C., Karaca, E., Scovell, J., Jhangiani, S.N., Akdemir, Z.C., Bainbridge, M., Yu, Y., Huff, C., Gibbs, R.A., Lupski, J.R., and Lamb, D.J. (2015). Whole-exome sequencing identifies novel homozygous mutation in npas 2 in family with nonobstructive azoospermia. Fertil Steril 104, 286-291.

Saraste, M., Sibbald, P.R., and Wittinghofer, A. (1990). The p-loop-a common motif in atp- and gtp-binding proteins. Trends Biochem Sci $15,430-434$.

Stahl, P.J., and Schlegel, P.N. (2012). Genetic evaluation of the azoospermic or severely oligozoospermic male. Curr Opin Obstet Gynecol 24, 221-228.

Tanaka, K., Miyamoto, N., Shouguchi-Miyata, J., and Ikeda, J.-E. (2006). Hfm1, the human homologue of yeast mer3, encodes a putative DNA helicase expressed specifically in germ-line cells. DNA Sequence 17, 242-246.

Wang, J., Zhang, W., Jiang, H., and Wu, B.-L. (2014). Mutations in HFM1 
in recessive primary ovarian insufficiency. $N$ Engl $J$ Med 370, 972-974.

Wang, K., Tang, D., Wang, M., Lu, J., Yu, H., Liu, J., Qian, B., Gong, Z.,

Wang, X., Chen, J., Gu, M., and Cheng, Z. (2009). Mer3 is required for normal meiotic crossover formation, but not for presynaptic alignment in rice. J Cell Sci 122, 2055-2063.

Wosnitzer, M.S. (2014). Genetic evaluation of male infertility. Transl Androl Urol 3, 17-26.

Open Access This article is distributed under the terms of the Creative Commons Attribution License which permits any use, distribution, and reproduction in any medium, provided the original author(s) and source are credited.

\section{SUPPORTING INFORMATION}

Figure S1 Information of HFM1 protein and sites of variants.

The supporting information is available online at life.scichina.com and link.springer.com. The supporting materials are published as submitted, without typesetting or editing. The responsibility for scientific accuracy and content remains entirely with the authors. 